moderately high titer of anti-cardiolipin antibodies (ACLA) or anti- $b_{2}$ glycoprotein-1 antibodies on more than one occasion.

Methods We identified 19 patients (17 females, 2 males), mean age of disease onset was 11.7 years (range 7-16 years). Patients were followed-up a median of 96 months (range 12-204 months).

Results Seven patients had only IgG ACLA, one had only IgM ACLA, two had both IgG and IgM ACLA, one had IgG and IgA ACLA, and one had all three ACLA's present. One patient had significantly positive LAC and IgM ACLA and four patients had positive results for both anti- $\beta 2$ glycoprotein and ACLA. Out of 17 patients, 11 had a diagnosis of Systemic Lupus Erythematosus (SLE) and 6 patients did not have any other connective tissue disease (CTD) diagnosis. On clinical presentation, 4 patients had headaches (HA), 4 had livedo reticularis, and 8 had both HA and livedo reticularis. At initial presentation, 1 patient had venous thrombosis, 1 had chorea nad one had both arterial and venous thrombosis with no previous CTD diagnosis. One patient with SLE presented with arterial thrombosis. In the follow-up period, one patient had a miscarriage and chose not to become pregnant again. All patients were placed on low-dose aspirin (ASA) $[81 \mathrm{mg} / \mathrm{d}]$ and hrdroxychloroquine (HCQ) if they had a diagnosis of SLE. Patients who had an event at presentation were treated with warfarin for 6 months and then switched to ASA alone after 6 months. There were no events noted in patients who were treated after their initial presentation to our clinic.

Conclusion This long-term follow-up shows that: 1) Livedo reticularis and HA are common initial presentations of APLS, but, 2) Prognosis has been very good with treatment with ASA and HCQ in the paediatric APLS group.

\section{FRI0173 MULTIPLE ANTIPHOSPHOLIPID-ANTIBODY-TESTS IMPROVE THE LABORATORY DIAGNOSTIC IN THE ANTIPHOSPHOLIPID SYNDROME}

${ }^{1} \mathrm{R}$ Wöhrle, ${ }^{2} \mathrm{~T}$ Matthias, ${ }^{1} \mathrm{M}$ Oppermann, ${ }^{1} \mathrm{~K}$ Helmke. ${ }^{1} \mathrm{~N}$. Med. Department, Municipal Hospital Munich-Bogenhausen, Munich; ${ }^{2}$ Aesku. Lab Diagnostika GmbH, Mikro Forum Ring 2, Wendelsheim, Germany

\subsection{6/annrheumdis-2001.242}

Background Examining patients with clinical features of the antiphospholipid-antibody-syndrome (APS), anticardiolipin antibodies (aCL) and lupus anticoagulant (LA) tests are widely performed. Unfortunately some patients with typical symptoms of APS (i.e. thromboembolic and ischaemic events, pregnancy losses) have negative test results in the commonly performed assays.

\section{Objectives}

Methods Using a new ELISA-test-system (Aesku.lab Diagnostika $\mathrm{GmbH}$, Wendelsheim, Germany) we examined sera of 102 patients (68 women, 34 men) with clinical symptoms of APS: 61 patients had a history of thromboembolic events, 44 of cerebrovascular insults, 6 women had pregnancy losses. The assay detects IgG- and IgM-class-antibodies against Phosphatidyl-Serine (PS), Phosphatidyl-Inositol (PI), Sphingomyelin (SM), Phosphatidyl-Ethanolamine (PE) and Phosphatidyl-Choline (PC) without cofactor and in combination with b2-glycoprotein I (b2-GP-I) respectively.

Results Sera of 50 patients were completely negative in the LAand aCL-tests, 17 patients had only low concentrations of aCLantibodies. Of this 67 patients with negative or borderline results in the commonly performed tests, 39 (58\%) were positive for other phospholipid-antibody-specificities, in 22 (33\%) the antibody-concentrations were even high. Antibodies which were tested positive in 10 or more patients are shown in the table, every "+" indicates one patient.

\begin{tabular}{lll} 
Abstract FRI0173 Table 1 & \\
\hline Antibody & Strong positive sera & Weak positive sera \\
\hline PS IgM without $\beta 2-$ GP-I & ++++++ & +++ \\
SM IgM without $\beta 2-$ GP-I & +++++++ & ++++ \\
PE IgM without $\beta 2$-GP-I & +++ & +++++++ \\
PC IgG without $\beta 2$-GP-I & ++++++++ & +++ \\
PC IgM without $\beta 2-$ GP-I & ++++++ & ++++ \\
\hline
\end{tabular}

Conclusion As shown in the table, most of the phospholipidantibody-specificities found in patients with negative or borderline results in LA- and aCL-tests are IgM-class antibodies independent from b2-glycoprotein I. Testing for these antibodies could be helpful for the diagnostic of patients with clinical features of the APS and negative results in the commonly performed tests, especially if there are no further risk factors for thromboembolic events or ischaemic insults.

\section{FRI0174 CATASTROPHIC ANTIPHOSPHOLIPID SYNDROME IN A PATIENT WITH SEVERE HYPERTENSION}

${ }^{1} S$ Rednic, 'L Damian, ${ }^{1} \mathrm{~L}$ Goga, ${ }^{2} \mathrm{M}$ Petrescu, ${ }^{3} \mathrm{~A}$ Cristea, ${ }^{1} \mathrm{HD}$ Bolosiu. ${ }^{1}$ Rheumatology; ${ }^{2}$ Pathology; ${ }^{3}$ Imunology, University of Medicine and Pharmacy, Cluj - Napoca, Romania

10.1136/annrheumdis-2001.243

Background The catastrophic antiphospholipid syndrome (CAPS) is an uncommon complication of the antiphospholipid syndrome (APS). It presents as acute multi-organ failure and more frequently involves the kidneys, lungs, gastrointestinal tract and the skin. CAPS is caused by widespread thrombosis of small and medium vessels.

Objectives

Methods Case report: We describe the case of a young female admitted with severe and accelerated hypertension. No evidence of systemic disease was observed at that time and the physical examination revealed extensive livedo reticularis. In subsequent years she also suffered several transient cerebrovascular accidents with minor neurological sequelae. The anticardiolipin antibodies (ACL) titers were usually and repeatedly extremely high. Renal angiography found normal proximal renal arteries. Renal biopsy showed ischaemic glomeruli without proliferative lesions, but with focal intimal fibrosis, thrombosis of the intrarenal vessels in the absence of vasculitis. The diagnostic of primary antiphospholipid syndrome was formulated. She was treated with antihypertensive drugs, anticoagulant and antiaggregant therapy, escalating doses of steroids, plasmapheresis.

Results Clinical course: Despite of this treatment she developed progressive multiorgan failure with malignant hypertension, renal failure, central nervous system manifestation (grand mal seizures, hemiparesis, amaurosis fugax, steady deterioration of consciousness) and gastrointestinal involvement (abdominal pain due to infarction or ischaemic ulceration of the bowel). She died as a result of a combination of renal and respiratory failure. Postmortem examination revealed extensive multiorgan arterial microthrombi. 